\title{
How to perform shear wave elastography. Part I
}

\author{
Giovanna Ferraioli', Richard G Barr ${ }^{2}$, André Farrokh ${ }^{3}$, Maija Radzina ${ }^{4}$, Xin Wu Cui ${ }^{5}$, Yi Dong ${ }^{6}$, \\ Laurence Rocher $^{7}$, Vito Cantisani ${ }^{8}$, Eleonora Polito ${ }^{8}$, Mirko D’Onofrio9, Davide Roccarina ${ }^{10,11}$, \\ Yasunobu Yamashita ${ }^{12}$, Manjiri K. Dighe ${ }^{13}$, Daniela Fodor ${ }^{14}$, Christoph F Dietrich ${ }^{15}$
}

${ }^{1}$ Department of Clinical, Surgical, Diagnostic and Pediatric Sciences, Medical School University of Pavia, Pavia, Italy, ${ }^{2}$ Northeastern Ohio Medical University, Rootstown, Ohio, USA, ${ }^{3}$ University Hospital Schleswig-Holstein, Campus Kiel, Department of Gynecology and Obstetrics, Germany, ${ }^{4}$ Diagnostic Radiology Institute, Paula Stradins Clinical University Hospital, Riga, Latvia, ${ }^{5}$ Sino-German Tongji-Caritas Research Center of Ultrasound in Medicine, Department of Medical Ultrasound, Tongji Hospital, Tongji Medical College, Huazhong University of Science and Technology, Wuhan, China, ${ }^{6}$ Department of Ultrasound, Zhongshan Hospital, Fudan University, Shanghai, China, ${ }^{7}$ Service de Radiologie, APHP Hôpitaux Paris Saclay, Hôpital Antoine Béclère, Clamart, France. Université Paris Saclay, Le Kremlin-Bicêtre, France, ${ }^{8}$ Department of Radiology, Oncology, Anatomo-Pathology, Sapienza-University of Rome, Rome, Italy, ${ }^{9}$ Department of Radiology, University Hospital G.B. Rossi, University of Verona, Italy, ${ }^{10}$ Sheila Sherlock Liver Unit and UCL Institute for Liver and Digestive Health, Royal Free Hospital, London, UK, ${ }^{11}$ SOD Medicina Interna ed Epatologia, Azienda Ospedaliero-Universitaria Careggi, Florence, Italy, ${ }^{12}$ Second Department of Internal Medicine, Wakayama Medical University, Wakayama, Japan, ${ }^{13}$ Department of Radiology, University of Washington, Seattle, USA, ${ }^{14} 2^{\text {nd }}$ Internal Medicine Department, "Iuliu Hatieganu" University of Medicine and Pharmacy, Cluj-Napoca, Romania, ${ }^{15}$ Department Allgemeine Innere Medizin (DAIM), Kliniken Hirslanden Beau Site, Salem und Permancence, Bern, Switzerland

\begin{abstract}
We recently introduced a series of papers describing how to do certain techniques. This article is the first part of a review of shear wave elastography (SWE). It reports the principles and interpretation of the technique and describes how to optimize it. Normal values, pitfalls and artefacts for the examination of liver, breast. thyroid and salivary gland with shear wave elastography are presented. The manuscript provides specific tips for applying SWE as part of a diagnostic US examination.
\end{abstract}

Keywords: ultrasound; elastography; elastometry; technique

\section{Introduction}

Ultrasound (US) elastography is a method to determine tissue stiffness. It is similar to palpation used in the physical examination. According to the current EFSUMB [1-5] and WFUMB guidelines [6-9] two types of US elastography can be defined: strain elastography (SE) and shear wave elastography (SWE). Strain uses an ap-

Received 30.01.2021 Accepted 15.03.2021

Med Ultrason

2022, Vol. 24, No 1, 95-106

Corresponding author: Prof. Christoph F. Dietrich

Department Allgemeine Innere Medizin (DAIM), Kliniken Hirslanden Beau Site,

Salem und Permancence, Bern, Switzerland

Phone: +41798347180

E-mail: c.f.dietrich@googlemail.com plied force either by palpation or an Acoustic Radiation Force Impulse (ARFI) method to create and receive information about tissue displacement associated with the elastic restoring forces in the tissue that act against shear deformation as a function of time and space to display biomechanical properties. This method has been previously described in detail $[10,11]$.

\section{Aim}

Two articles on "how to perform strain imaging techniques" have been recently published using conventional [10] and endoscopic US elastography [11]. This article is the first part of a review of SWE applied to several organs. It describes how to optimize the examination technique, discussing normal values, pitfalls and artefacts for the examination of the liver, breast. thyroid and salivary glands. The manuscript provides more specific tips for applying SWE as part of a diagnostic US examination. 
Shear wave based elastography - how does it work?

SWE techniques include vibration controlled transient elastography (VCTE) and ARFI based techniques. The shear waves are generated by a body-surface vibration, as in VCTE, or by the push-pulse of a focused US beam, as in ARFI techniques. In VCTE, a body-surface vibration creates a shear wave, which then travels to the organ of interest. The frequency of the vibration is controlled $(50 \mathrm{~Hz})$, as are its shape and amplitude. VCTE is implemented on the Fibroscan ${ }^{\circledR}$, which is a dedicated device that does not display an anatomical image. In ARFI-based techniques, the shear waves are generated directly in the tissue. A convex or linear transducer transmits focused US pulses (also known as a push pulses or ARFI) that generate shear waves. The pulses are repeated several times over a short period of time, and the shear waves generated travel at a much slower rate than US. B-mode tracking pulses are used to detect the propagation velocity of the shear wave [12] by measuring the difference in arrival time (time lag) between two points at known distances apart from each other $[1,6,13]$. Such push pulses generate much slower shear waves off-axis [14]. ARFI-based techniques include point shear-wave elastography (pSWE) and multidimensional SWE (2DSWE, 3D-SWE). pSWE measures the stiffness at the focal $\left(\sim 1 \mathrm{~cm}^{3}\right)$ point in the tissue whereas with 2D-SWE the stiffness is measured over a much larger area and a color-coded image of the qualitative elastic properties is displayed on the monitor of the US system $[3,4]$.

\section{Shear wave speed}

The shear wave speed (SWS) is almost one thousand times lower than the velocity of US in soft tissues, the shear waves attenuate very rapidly and some do not propagate in the simple fluids [14]. The shear wave propagates faster in stiffer tissue than in softer tissue. The expected SWS in the liver in normal and pathologic states is typically in the range 0.7 to $5.0 \mathrm{~m} / \mathrm{s}(1.5 \mathrm{kPa}$ to $75 \mathrm{kPa})$. For breast cancers it can be up to $10 \mathrm{~m} / \mathrm{s}(300 \mathrm{kPa})$ and even higher for normal tendons. Pathology in any tissue often creates changes in tissue stiffness making elastography a method to characterize pathological changes. SWS values may vary depending on the vendor; therefore, vendor specific cut-off values may be necessary.

\section{Differences of equipment}

Different equipment may give different values of stiffness within the same tissue in the same patient. This is because the measured values of SWS will vary with a number of system factors, in particular shear-wave vibration mean frequency and bandwidth. In addition, measurement bias may occur due to the algorithm employed to calculate relative shear wave arrival time and speed.

\section{$\mathrm{kPa}$ or can they change to $\mathrm{m} / \mathrm{s}$ ?}

The propagating speed [12] of the generated shear wave is reported in meters per second $(\mathrm{m} / \mathrm{s})$ but can also be converted to Young's modulus values in kilopascals $(\mathrm{kPa})$ by applying the formula $\mathrm{E}=3 \rho \mathrm{Vs}^{2}$, where $\mathrm{E}$ is tissue elasticity, Vs is the shear wave speed, and $\rho$ is the density of tissue in $\mathrm{kg} / \mathrm{m}^{3}$, and making some assumptions $[1-3,6]$. One main reason why it is preferable to report results in units of $\mathrm{ms}^{-1}$ rather than $\mathrm{kPa}$ is the fact that the SWS is measured by the scanner in $\mathrm{ms}^{-1}$. However, mainly for liver application, the units of the Young's modulus are largely used, as many clinicians are familiar with them.

\section{Angle of insonation}

The angle of insonation has a significant influence on the measurement, which is of importance when a curved transducer is used [15]. The shear waves are generated perpendicular to the ARFI push pulse therefore the Bmode tracking must be in the same angle to accurately estimate the SWS.

\section{Region of interest}

The ROI should be positioned so that the push pulse is generated perpendicular to the center of the transducer surface. For more information about the technology we also refer to the recently published guidelines on elastography $[1,7,15,16]$.

Does the size and/or the shape of the ROI influences measurements?

The size of the ROI depends on the tissue being evaluated. Even though a larger ROI would give SWE information over a larger amount of tissue, it risks the inclusion of artifacts particularly in heterogeneous masses. By using two different 2D-SWE US systems, it has been shown that, for the assessment of breast lesions, a small round ROI (approximately $2 \mathrm{~mm}$ in diameter) placed over the stiffest area of the lesion was more accurate than a larger ROI manually drawn along the margin of the lesion [17]. In another study that assessed the influence on the accuracy of 2D-SWE in evaluating breast lesions by using three different ROI size (1, 2 and $3 \mathrm{~mm})$, the diagnostic accuracy was not affected by changing the ROI size [18]. In general, malignant lesions are heterogeneous in stiffness and using the area of highest stiffness is more accurate in characterizing the lesion. However, for homogenous tissue like liver, a larger ROI can average the stiffness over a larger area of tissue.

In ex vivo study involving porcine muscle, a significant increase of SWS $(p<0.001)$ was observed for larger ROI widths. In this animal model, the SWS was also influenced by several other factors, including probe frequency, applied pressure, muscle orientation, different machine settings, and placement depth [19]. 


\section{Artefacts}

SWE images are reconstructed using time-of-flight based images. In heterogeneous tissues these algorithms might introduce a variety of artifacts. One of them is SWE under- and overestimation from reflections at stiffness interfaces. Reflected waves violate the assumption of a single direction of propagation, leading to artifacts in SWE images [20]. To avoid this, directional filters had been applied [20,21]. By separating the forward and backward components, it is possible to almost entirely remove the reflected wave [21]. It is highly recommended in transient shear wave applications to avoid reflection artifacts. For liver assessment, common artifacts include reverberation from the liver capsule, respiratory/cardiac motion and vessel pulsation/loss of the SWE signal (fig 1). The penetration of the US beam can also generate artifacts since consistent elasticity estimates cannot be obtained in the far field due to attenuation of the ARFI pulse. The most consistent estimates are generally obtained near the focus zone of the ARFI pulse, where the largest displacements is generated by the push pulses [22]. A detailed analysis of all the artifacts is out of the scope of this review article and can be found elsewhere [22-24].

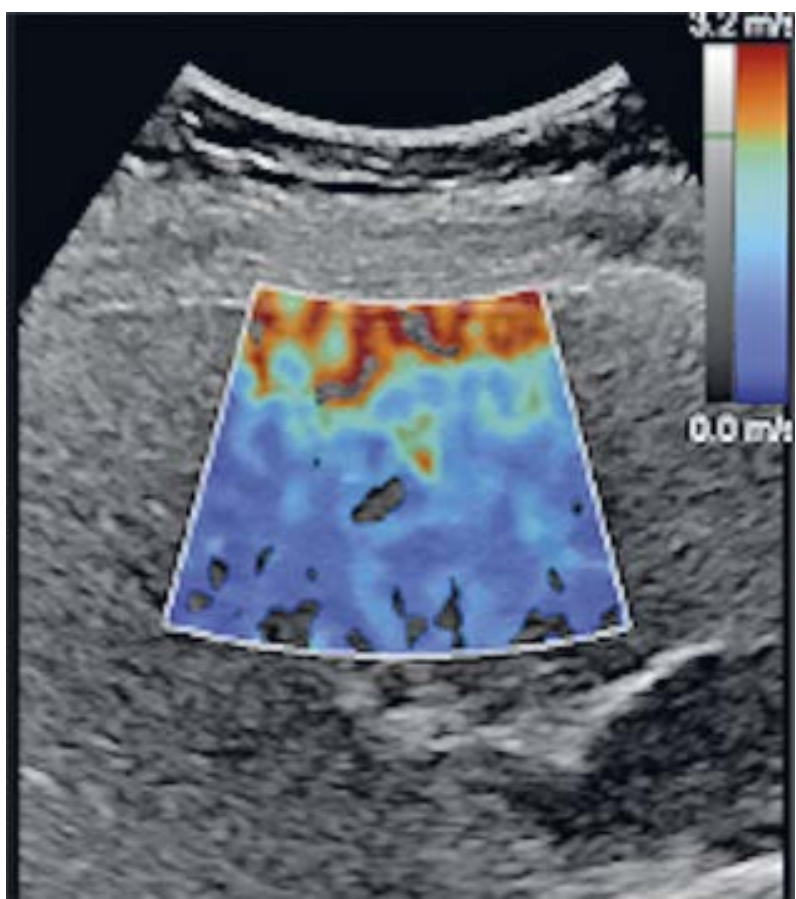

Fig 1. Example of the reverberation artifact from the liver capsule in SWE. The red and teal areas are the artifacts; the blue areas are the accurate stiffness measurements. Note that in p-SWE a color map is not provided, so it is critical to place the ROI box $1.5-2 \mathrm{~cm}$ below the liver capsule. Whereas in 2DSWE the artifact can be identified on the color map and be avoided.

\section{Liver}

\section{Main objective, clinical value}

The liver is an important target organ for the use of elastography; stiffness correlates with the degree of fibrosis and indirectly with portal hypertension and the risk of developing hepatocellular carcinoma. Due to the large overlap between stiffness values, guidelines do not recommend the use of SWE to differentiate benign and malignant focal liver lesions [15,25-28].

The most important clinical management may be summarized as follows:

1. SWE values within the normal range can rule out compensated advanced chronic liver disease (cACLD) when in agreement with the clinical and laboratory data.

2. SWE technologies perform best to rule out cirrhosis.

3. SWE technologies can be used as first line assessment for the severity of liver fibrosis but are much less reliable in differentiating intermediate stages of fibrosis.

4. An interquartile range/median (IQR/M) $\leq 30 \%$ with measurements taken in $\mathrm{kPa}$ or $<15 \%$ when taken in $\mathrm{m} / \mathrm{s}$ is the most important reliability criterion [29].

\section{"Knobology" \\ Prerequisites}

The user should always refer to the manufacturer's recommendations for a good quality measurement. The parameters that should be taken into account vary from one manufacturer to another and include judgment of the signal-to-noise ratio or the stability of the signal over time (2D-SWE acquisitions). Several manufacturers have developed quality criteria for either pSWE or 2DSWE techniques. The users must always refer to them when they are available.

\section{Transient elastography: probe selection}

In transient elastography (TE) three different probes are available (S, M and XL probes). The $\mathrm{S}$ probe is used in children with a thoracic belt $<75 \mathrm{~cm}$ whereas the XL probe is dedicated to overweight/obese subjects with more reliable results as compared with the $\mathrm{M}$ probe. The XL probe must be used when the skin-to-liver capsule distance is higher than $25 \mathrm{~mm}$. Limiting factors for the $\mathrm{XL}$ probe are a skin-to-liver capsule distance $>3.4 \mathrm{~cm}$ and extreme obesity $\left(B M I>40 \mathrm{~kg} / \mathrm{m}^{2}\right)$ [3,4,28]. Values obtained with XL probe are usually lower than with the $\mathrm{M}$ probe, therefore no recommendation on the cut-offs to be used can be given.

ARFI-basedtechniques: transducer(frequency) selection

In adults, the convex transducer is used for performing the elastography studies, whereas in children the choice of the probe, either the linear or the curvilinear one, depends on the body habitus and age. Generally, the 
same rule used for the choice between the two probes for the B-mode image of the liver applies also to the assessment of liver stiffness (LS) in children. However, it should be kept in mind that the difference in frequency between the two probes gives different readings in the same subject. In phantom studies, it has been shown that the readings with the higher frequency of the linear transducer are higher than those obtained with the convex transducer. Moreover, in children the acquisition could be more challenging due to the lack of cooperation and this could affect the feasibility of the technique [30,31].

Description of (other) parameters

The strength of the push-pulse is higher in the center of the transducer, thus the sampling should be done in the central area of the image, whereas the sampling at the edge should be avoided.

The influence of depth on the estimation of the elastic properties is not negligible [32]. The acoustic push pulse is progressively attenuated as it traverses the tissue. The results with the lowest variability are obtained at a depth of $4-5 \mathrm{~cm}$ from the skin surface [33]. The attenuation is higher in stiffer liver, thus in cirrhotic or steatotic patients, measurements are more variable [15]. The ROI box should be perpendicular to the transducer.

Region of interest (ROI) size, shape, others

The region of interest should be in between $2-6 \mathrm{~cm}$ below the liver capsule.

In pSWE the size of the region of interest (ROI) is small and fixed by the manufacturer because the technique assesses the stiffness at a single location by using a sequence of push-pulses, generally up to five.

In 2D-SWE the size of the ROI is user-adjustable and can theoretically be as large as the ARFI FOV image. However, the larger the ROI the higher the risk of including artifacts. Thus, generally the ROI's size in SWE technique may influence the quality of the elastogram. Following EFSUMB guidelines and recommendations, we suggest using an ROI of $2.5 \times 2.5 \mathrm{~cm}$ in size $[3,4]$. Many vendors have quality or confidence maps, which help to identify and avoid artifacts [15].

\section{Position of the transducer}

The measurements should be performed through the intercostal space rather than the subcostal approach yielding the highest intra- and interobserver agreement [15,34-36].

\section{Description of quality parameters}

The most important criterion for a measurement of good quality seems an IQR/M $\leq 30 \%$ when the results are reported in Young's modulus [29]. This ratio, in fact, is a measure of the variability between consecutive acquisitions, and studies have reported a decrease in accuracy when this criterion is not fulfilled [37-41]. For measure- ment reported in $\mathrm{m} / \mathrm{s}$ the IQR/M should be $\leq 15 \%$ because the conversion between the two is not linear [29].

Pre-compression

Pre-compression should be avoided.

How many measurements?

Based on literature data, for the pSWE technique the EFSUMB and WFUMB guidelines have recommended to use the median value of 10 acquisitions [3,4,9]. However, some studies have shown that the accuracy does not decrease when fewer acquisitions (up to five) are obtained [38-40,42]. For 2D-SWE, the EFSUMB updated guidelines have recommended to obtain at least three acquisitions $[3,4]$. The updated WFUMB and SRU guidelines are more cautious and have suggested five acquisitions when a quality factor is available $[9,43]$. The higher number of acquisitions suggested by the WFUMB updated guidelines may give a better estimation of the variability assessed through the calculation of the IQR/M ratio.

\section{Reproducibility}

The intra-observer reproducibility of VCTE [44-46], pSWE [34,36,47-49] and 2D-SWE [50-52] for LS assessment is excellent with ICC above 0.90 .

\section{How to use shear wave elastography}

The transducer should be positioned in an intercostal space; perpendicular to the liver in both superior/inferior and right/left planes, avoiding the ribs or the lung artifacts. As the SWS is calculated based on B-mode, the quality of the B-mode US image affects the quality of the SWE acquisitions. The most common limitations encountered with US, i.e. poor acoustic window, limited penetration, and rib or lung shadowing, may influence both the feasibility and the performance of the SWE techniques. Some of these limitations can be avoided, thus the operator should obtain an optimal scan of the liver before launching the acquisition. The perpendicular position of the transducer can be assessed by looking at the liver capsule that appears as a sharp white line, parallel to the transducer's line (fig 2). Motion of the probe or of the patient affects the quality of the measurement as well. The patient should breathe normally while the operator is searching for the best acoustic window and for the best area of liver parenchyma where the sample box will be positioned. This area should be homogeneous, i.e., free of vessels or ligaments. Before launching the acquisition, the operator asks the patient to hold the breath in a neutral position without performing a Valsalva's maneuver for the few seconds needed for the acquisition [15]. Special applications in pediatric patients are discussed elsewhere [30,31,53].

\section{Tips and tricks}

Depth as assessed by the skin-to-liver capsule distance may influence the SWS values assessed by all 


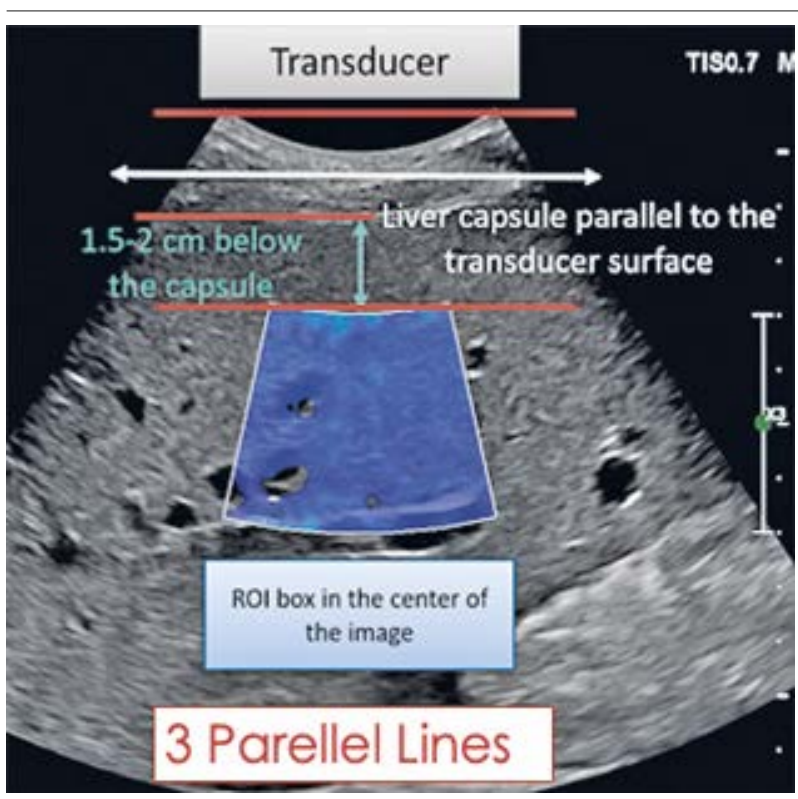

Fig 2. Figure demonstrating the positioning of the liver capsule and FOV box in liver stiffness assessment. The transducer, liver capsule and top of the FOV box should be parallel lines. The liver capsule should be a sharp echogenic line.

SWE-techniques. Due to the attenuation of the US beam, the depth for reliable measurements is up to $7 \mathrm{~cm}$ in most systems; measurements performed deeper have a lower signal/noise ratio. Using a deep abdominal probe may allow for measurements at a greater depth in high BMI patients. In ARFI techniques, the US beam that generates the shear waves is also attenuated by the interaction with the tissue that it traverses thus, its strength is inversely related to the depth; this attenuation is higher in cases of liver steatosis or severe fibrosis. Due to these factors, measurements in patients with significant liver steatosis or severe fibrosis could have a higher rate of unreliable results or failures. This is also true in obese patients with thick subcutaneous tissue due to higher attenuation of the US beam in the near field. In staging liver fibrosis, Metavir-derived cutoff values are system-specific and could not be applied interchangeably across different US systems. A recent study has shown that the agreement between LS measurements obtained with different US systems is good to excellent; however, the difference between values was higher than two $\mathrm{kPa}$, assigning the patient to different stages of liver fibrosis [54]. Because the overlap of LS values between METAVIR-derived scores is as large if not larger than the difference between vendors, the updated SRU consensus advises that separate cut-off values for each vendor are not required when determining the likelihood for cACLD [29]. The SWE values might be overestimated in certain diseases, e.g. sinusoidal obstruction syndrome due to congestion [55].

\section{Normal reference values}

For all equipment, a SWE measurement within the normal range, in a subject without other clinical or laboratory evidence of liver disease, may exclude significant liver fibrosis with a high degree of certainty. For both VCTE and ARFI-based techniques, there is consensus in considering that values $\leq 5 \mathrm{kPa}(1.3 \mathrm{~m} / \mathrm{s})$ have high probability of being normal $[29,56]$.

\section{What to avoid?}

Confounding factors that may lead to an increase of LS independently from liver fibrosis have been listed elsewhere [57-59]. Briefly, eating may increase the stiffness of the liver, thus measurements are performed in the fasting status of at least 4 hours. LS does not necessarily reflect liver fibrosis, but can reflect many other physiological or pathological conditions including hepatic inflammation (elevated transaminase level) [60-63], obstructive cholestasis [64], neoplastic and other infiltration of the liver and hepatic congestion $[65,66]$. Recently, it has been reported that portal vein thrombosis is also a confounder [67]. On the other hand, SWE can play a role in cases of liver congestion due to right-sided heart failure, congenital heart diseases or valvular diseases as well as in the hepatic sinusoidal obstruction syndrome or in the Budd-Chiari syndrome [31,68].

\section{Specific artifacts}

Measurements should be performed at least $1-2 \mathrm{~cm}$ below the liver capsule to avoid reverberation artifacts. However, when using 2D-SWE with a quality map the measurement can be taken closer to the liver capsule as the artifact can be visualized and avoided. This is helpful in high BMI or steatotic patients since the reverberation artifact in these patients can be as small as $5 \mathrm{~mm}$ and visualizing the artifact on 2D-SWE may help with placing the ROI closer to the liver surface and still avoid the reverberation artifact.

\section{Breast}

\section{Main objective, clinical value}

Various studies have shown that malignant and benign breast tumours differ significantly in their elasticity [7,69-75]. Benign alterations tend to be softer than malignant lesions. This fact forms the basis for the use of elastography to differentiate between different breast tumors. SWE is a new method introduced in 2009 and, unlike strain elastography, allows quantitative measurement of tissue stiffness. SWE is not only capable of the differentiation between benign and malignant tumours, but can also be used for therapy monitoring under neoadjuvant chemotherapy [76-79]. Recently, the fifth edition of the ACR BI-RADS Atlas 2013 incorporated elasticity 
assessment of breast lesions as one of the associated features of ultrasound [80].

\section{"Knobology"}

Transducer (frequency) selection

A standard $5 \mathrm{~cm}$ wide linear transducer is very well suited to perform SWE of breast lesions. Depending on vendor, transducers of $9 \mathrm{MHz}$ to $18 \mathrm{MHz}$ are SWE enabled. Before the elastography mode is activated, a highquality B-mode image must first be set, because the elastogram is derived from it. It is recommended to use higher US frequencies in the assessment of superficial breast lesions and lower frequencies for better depth penetration for lesions located deep inside the breast. The operator must be aware that lower frequencies result in a lower spatial resolution. The US probe must be placed perpendicular on the skin of breast directly above the lesion with enough contact to the breast tissue to obtain a good Bmode image while avoiding excessive pre-compression. Pre-compression can be recognized when fatty tissue that should normally appear blue (soft) on the color map has a different color (fig 3). The examiner must not move the US probe while the elastogram is being obtained.

Region of interest (ROI) size

There are several approaches in setting the ROI size. One way is to use a small ROI placed at the site of the stiffest area within the mass or within $3 \mathrm{~cm}$ surrounding the mass. Another way is to use larger ROIs that cover the entire lesion. No general standard is given within published guidelines. A recent paper evaluating 154 breast lesions came to the conclusion that a small ROI measuring the mean or maximum stiffness value is superior to medium sized or large sized ROI in distinguishing between benign and malignant lesions [17]. Regardless of the size of the ROI, it has been shown that minimum stiffness value is the least significant and should therefore not be used.

\section{Description of quality parameters}

Some vendors provide a quality map, which is a color-coded map that can be superimposed on the Bmode US image and provides information of the quality of the shear wave propagation and the image quality. Even green distributions indicate a high quality elastogram, whereas yellow or red areas should not be used for assessment [81].

\section{Pre-compression}

Pre-compression is an important factor of influence changing the appearance of a lesion in the elastogram. If too much pre-compression were applied, the lesion would appear stiffer than it really is [82]. Therefore, the recommendation is to apply a large amount of gel and then place the US transducer on the breast. The subcutaneous fatty tissue should appear in dark blue. If it appears

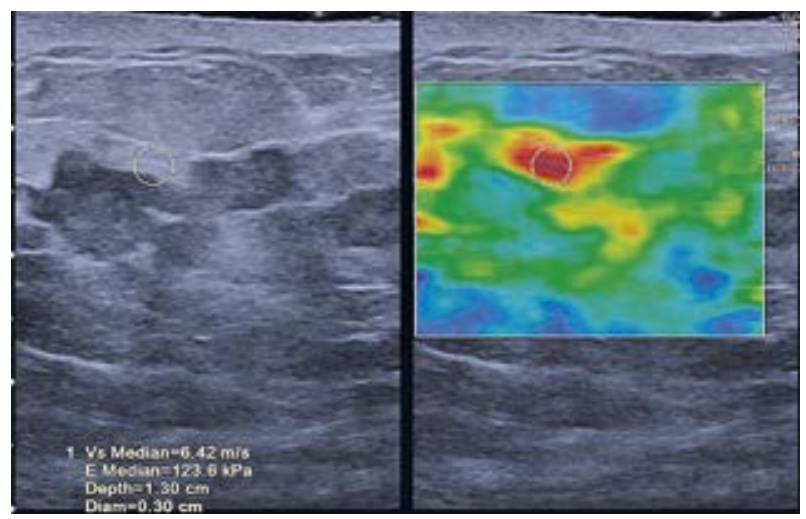

Fig 3. 2D-SWE of an invasive ductal cancer. The irregular hypoechoic lesion is the malignancy. Note that the skin surface is parallel to the transducer, there is a small amount of coupling gel between the transducer and skin confirming minimal precompression and that the area of highest stiffness is just adjacent to the lesion.

in green or even red than too much pre-compression is used and needs to be corrected.

Checking reproducibility

To assess the quality and reproducibility of the elastography image, place the probe and hold it still while the elastogram builds superimposed on the B-mode image. Wait 5-10 seconds until the elastograms shows a consistent and permanent color pattern. Also check that the fatty tissue is displayed soft, which indicates that not too much pre-compression is applied. Then freeze the picture and proceed with the measurements within the elastogram.

\section{How to use shear wave elastography}

The basic recommendations about performing SWE as described above also apply to the use in breast tumours. SWE should be used as a lesion-based adjunct to conventional B-mode imaging using all ACR BI-RADS criteria giving the examiner more information in order to make a final assessment. It should not be used as a screening tool without a lesion. However, if a palpable lesion is present with no B-mode findings elastography may identify an isoechoic lesion. A quantitative assessment using the mean or maximum stiffness values can be used. Alternatively, the color pattern of the elastogram can be analyzed by using different color pattern scores $[83,84]$.

\section{Artifacts}

There is a well-documented artifact with SWE in malignant lesions. They may appear as soft lesions even though they are very stiff. This artifact is not infrequent and can be recognized by evaluating the quality map. In these false negative cases the quality map usually will confirm that there are poor shear wave and the results should not be used. These cases of false negative lesions on SWE are always true positives on SE. Therefore, the 
combination of SE and SWE will improve diagnostic accuracy $[71,72,85]$.

Tips and tricks

- Use ultrasound frequency according to lesion localization (depth);

- Use small ROI and mean or maximum stiffness values for assessment;

- Change the clinical procedure for BI-RADS 4a and BI-RADS 3 lesions according to SWE measurements;

- If the stiff rim sign occurs measure within the stiff rim and not inside the uncolored lesions center.

Normal reference values

Normal fatty tissue: mean stiffness values $5-10 \mathrm{kPa}$ $(1.3-1.8 \mathrm{~m} / \mathrm{s})$; breast parenchyma: mean stiffness values $30-50 \mathrm{kPa}(3.1-4.1 \mathrm{~m} / \mathrm{s})$ [86].

Differentiating between benign and malignant lesions the following cut-off values are reported: maximum stiffness values $33.3-80 \mathrm{kPa},(3.3-5.0 \mathrm{~m} / \mathrm{s})$; mean stiffness values $46.7-93.8 \mathrm{kPa},(4.0-5.6 \mathrm{~m} / \mathrm{s})$.

What to avoid?

During examination it should be avoided the precompression by checking the stiffness of fatty tissue which should be in the normal range, the use of minimum stiffness values for the differentiation between benign and malignant breast lesions and measurements in areas of poor quality on the quality map.

\section{Thyroid}

\section{Main objective, clinical value}

Despite fine needle aspiration (FNA) being the gold standard in the diagnosis of thyroid neoplasms, US has a paramount role in the diagnostic process. Thyroid nodules are indeed present in almost $50 \%$ of the population and so performing cytology on one or multiple targets on each patient is not feasible; the detection of certain suspect features on B-mode US is then fundamental in deciding which nodules should be assessed with FNA $[87,88]$. SWE is a quick, readily available tool, and effective in increasing US sensitivity in the detection of thyroid neoplasms [89].

\section{"Knobology"}

Prerequisites

It is recommended to verify the manufacturer's instructions about quality parameters. No patient preparation is required; patient has to lie down in supine position with a pillow or a towel used to extend the patient's neck [2].

Select an appropriate transducer and frequency selection

Select a high-end linear transducer, usually 7 to 18 $\mathrm{MHz}$ [90].

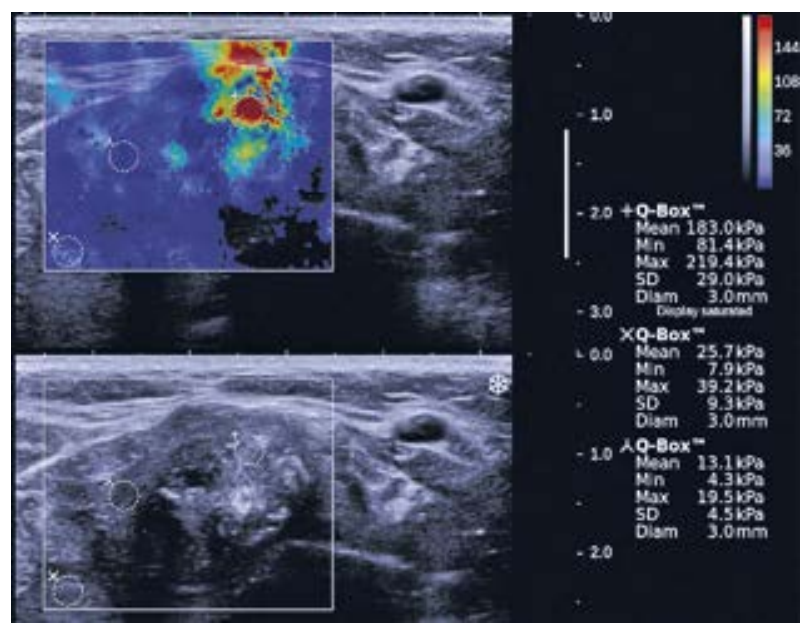

Fig 4. SWE examination of a thyroid with several nodules. The ROI is placed in each nodule to avoid including normal thyroid or other structures. In this case the more central nodule with a mean stiffness value of $183 \mathrm{kPa}$ was a papillary carcinoma. The other two nodules were benign.

Region of interest (ROI) size, shape, others

The sample box features depend on the SWE method being used. In pSWE the small fixed-size ROI should be completely included within the nodule. In 2D-SWE the box should be large enough to include the whole nodule, avoiding nearby vessels or gland areas with cystic or fibrotic changes (fig 4) [5,91].

Description of quality parameters

For pSWE 3 to 10 measurements should be acquired at the same location and the average of these should be calculated. For 2D-SWE at least three measurements must be performed [5,91].

\section{Pre-compression}

An abundant quantity of gel should be used to avoid pre-compression, since it may alter tissue elastic modulus thus causing artifacts. The operator places the transducer perpendicular to the target nodule without pressure, maintaining only slight contact with the skin. A manufacturer quality control tool should be used if available [92].

\section{Normal reference values}

Normal values may vary depending on the manufacturer; however, guidelines suggest that benign nodules show a mean elasticity of $15.3-28 \mathrm{kPa}$ [5]. Recent studies state that the optimal cut-off between benign and malignant nodules is $34.5-37.5 \mathrm{kPa}[93,94]$.

What to avoid?

As stated before, it is important to avoid compression artifacts, which may jeopardize measurement's accuracy and reliability. Certain neck morphologies may be challenging when correctly placing the probe perpendicular to the target nodule; previous neck surgery and subsequent fibrosis may as well represent an obstacle [2]. 


\section{Salivary glands}

\section{Main objective, clinical value}

Salivary glands are readily accessible to high resolution US, which is the initial imaging modality when clinically indicated. SWE is useful for the assessment of diffuse diseases, such as Sjögren syndrome, parotitis in pediatric patients or damage due to irradiation [95-97]. As for the evaluation of focal lesions, a substantial overlap of stiffness values has been reported [94]. A multiparametric approach to allow a better differentiation between benign and malignant lesions has been suggested [90].

\section{"Knobology"}

Prerequisites

Before starting shear wave measurement, it is recommended to verify the quality of the shear wave generation by referring to the manufacturer's quality parameters.

Transducer (frequency) selection

SWE is performed with a high-frequency linear transducer, typically 7 to $12 \mathrm{MHz}$, with patient lying in the supine position with a pillow or a towel used to extend the patient's neck $[90,98]$.

Region of interest (ROI) size, shape, others

The sample box should be positioned in a region of the gland free of vessels or cystic or fibrotic transformation, and between 1 to $2 \mathrm{~cm}$ from the anterior glandular contour.

\section{Description of quality parameters}

For pSWE, the fixed ROI should be placed at the point of interest. Three to 10 measurements should be acquired and the median value calculated. For 2D-SWE, because a larger FOV is available, at least three measurements must be performed [5].

\section{Pre-compression}

Pre-compression should be avoided.

\section{Tips and tricks}

For obtaining a prompt and reliable SWE acquisition, it is recommended to hold the transducer perpendicular to the plane being explored, avoiding any movement of the transducer or of the patient when the acquisition has been launched. A sufficient quantity of gel has to be used and minimal pressure should be applied to avoid precompression because it can alter tissue elastic modules and produce artifacts. False negative cases do occur and if the B-mode findings are suggestive of a malignancy a biopsy should not be cancelled based on elastography. When there are several similar nodules, SWE can be used to select the stiffest lesion for sampling.

\section{Normal reference values}

Literature data suggest that the mean stiffness of the salivary glands is quite uniform (approximately $11 \pm 3$ $\mathrm{kPa}$ ) (fig 5) [99].

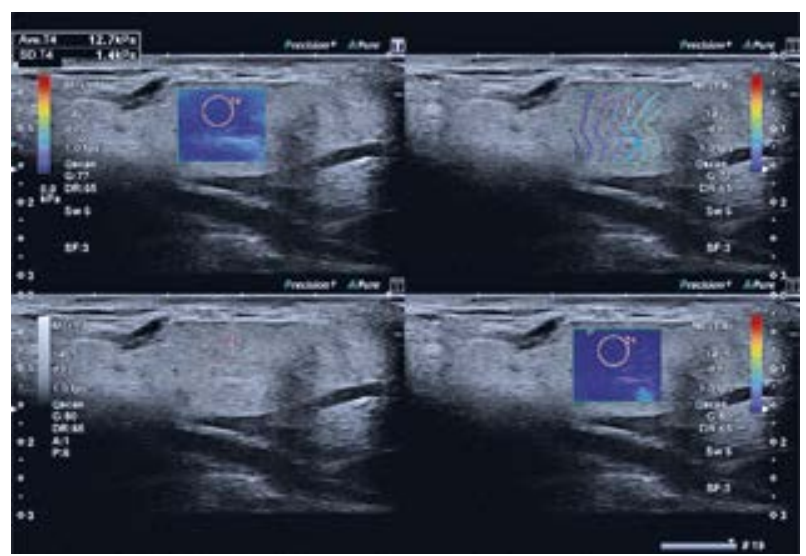

Fig 5. 2D-SWE of a normal parotid gland.

\section{What to avoid}

As explained for other organs, artifacts should be limited for a prompt and reliable elastography acquisition. The interpretation of a 2D-SWE elastogram is operator dependent and the choice of an adequate ROI is challenging due to the multiplicity and complexity of the structures in the neck region.

\section{Specific artifacts}

The reliability of the measurement may be affected by some artifacts that may arise in the region of the neck, generally due to the proximity to the skin, to the osseous plane (ramus of mandible) or to the eventual presence of focal convex bulge of the skin, which generates local inhomogeneity and falsify the real elasticity of the tissue. Some very stiff cancers show a circular stiff area in the surroundings of the actual lesion. This artifact is called the "stiff rim sign". In 3D SWE there is a similar sign in the c-plane called the "crater sign" [100]. The reason for these artifacts is still under discussion but it may indicate a very low shear wave amplitude within the cancer due to attenuation of US energy resulting in no colour coded information in the elastogram [101]. Furthermore, the "stiff ring sign" and the "crater sign" can be used as predictors of malignancy [102].

Acknowledgement. The authors thank the Bad Mergentheimer Leberzentrum e.V. for support.

\section{References}

1. Bamber J, Cosgrove D, Dietrich CF, et al. EFSUMB guidelines and recommendations on the clinical use of ultrasound elastography. Part 1: Basic principles and technology. U1traschall Med 2013;34:169-184.

2. Cosgrove D, Piscaglia F, Bamber J, et al. EFSUMB guidelines and recommendations on the clinical use of ultrasound elastography. Part 2: Clinical applications. Ultraschall Med 2013;34:238-253. 
3. Dietrich CF, Bamber J, Berzigotti A, et al. EFSUMB Guidelines and Recommendations on the Clinical Use of Liver Ultrasound Elastography, Update 2017 (Long Version). Ultraschall Med 2017;38:e16-e47.

4. Dietrich CF, Bamber J, Berzigotti A, et al. EFSUMB Guidelines and Recommendations on the Clinical Use of Liver Ultrasound Elastography, Update 2017 (Short Version). Ultraschall Med 2017;38:377-394.

5. Săftoiu A, Gilja OH, Sidhu PS, et al. The EFSUMB Guidelines and Recommendations for the Clinical Practice of Elastography in Non-Hepatic Applications: Update 2018. Ultraschall Med 2019;40:425-453.

6. Shiina T, Nightingale KR, Palmeri ML, et al. WFUMB guidelines and recommendations for clinical use of ultrasound elastography: Part 1: basic principles and terminology. Ultrasound Med Biol 2015;41:1126-1147.

7. Barr RG, Nakashima K, Amy D, et al. WFUMB guidelines and recommendations for clinical use of ultrasound elastography: Part 2: breast. Ultrasound Med Biol 2015;41:11481160.

8. Ferraioli G, Filice C, Castera L, et al. WFUMB guidelines and recommendations for clinical use of ultrasound elastography: Part 3: liver. Ultrasound Med Biol 2015;41:11611179.

9. Ferraioli G, Wong VW, Castera L, et al. Liver Ultrasound Elastography: An Update to the World Federation for Ultrasound in Medicine and Biology Guidelines and Recommendations. Ultrasound Med Biol 2018;44:2419-2440.

10. Dietrich CF, Barr RG, Farrokh A, et al. Strain Elastography - How To Do It? Ultrasound Int Open 2017;3:E137-E149.

11. Dietrich CF, Bibby E, Jenssen C, Saftoiu A, Iglesias-Garcia J, Havre RF. EUS elastography: How to do it? Endose Ultrasound 2018;7:20-28.

12. Dietrich C, Working Group on Sonography D. [Report of the Working Group on Sonography DGVS]. Ultraschall Med 2003;24:3-4.

13. Dietrich CF, Dong Y. Shear wave elastography with a new reliability indicator. J Ultrason 2016;16:281-287.

14. Sarvazyan AP, Rudenko OV, Swanson SD, Fowlkes JB, Emelianov SY. Shear wave elasticity imaging: a new ultrasonic technology of medical diagnostics. Ultrasound Med Biol 1998;24:1419-1435.

15. Barr RG, Ferraioli G, Palmeri ML, et al. Elastography Assessment of Liver Fibrosis: Society of Radiologists in Ultrasound Consensus Conference Statement. Radiology 2015;276:845-861.

16. Barr RG, Cosgrove D, Brock M, et al. WFUMB Guidelines and Recommendations on the Clinical Use of Ultrasound Elastography: Part 5. Prostate. Ultrasound Med Biol 2017;43:27-48.

17. Moon JH, Hwang JY, Park JS, Koh SH, Park SY. Impact of region of interest (ROI) size on the diagnostic performance of shear wave elastography in differentiating solid breast lesions. Acta Radiol 2018;59:657-663.

18. Skerl K, Vinnicombe S, Giannotti E, Thomson K, Evans A. Influence of region of interest size and ultrasound lesion size on the performance of $2 \mathrm{D}$ shear wave elastography
(SWE) in solid breast masses. Clin Radiol 2015;70:14211427.

19. Rominger MB, Kälin P, Mastalerz M, et al. Influencing Factors of 2D Shear Wave Elastography of the Muscle - An Ex Vivo Animal Study. Ultrasound Int Open 2018;4:E54-e60.

20. Lipman SL, Rouze NC, Palmeri ML, Nightingale KR. Evaluating the Improvement in Shear Wave Speed Image Quality Using Multidimensional Directional Filters in the Presence of Reflection Artifacts. IEEE Trans Ultrason Ferroelectr Freq Control 2016;63:1049-1063.

21. Deffieux T, Gennisson JL, Bercoff J, Tanter M. On the effects of reflected waves in transient shear wave elastography. IEEE Trans Ultrason Ferroelectr Freq Control 2011;58:2032-2035.

22. Bruce M, Kolokythas O, Ferraioli G, Filice C, O'Donnell M. Limitations and artifacts in shear-wave elastography of the liver. Biomed Eng Lett 2017;7:81-89.

23. Bouchet P, Gennisson JL, Podda A, Alilet M, Carrié M, Aubry S. Artifacts and Technical Restrictions in 2D Shear Wave Elastography. Ultraschall Med 2020;41:267277.

24. Dubinsky TJ, Shah HU, Erpelding TN, Sannananja B, Sonneborn R, Zhang M. Propagation Imaging in the Demonstration of Common Shear Wave Artifacts. J Ultrasound Med 2019;38:1611-1616.

25. Yu H, Wilson SR. Differentiation of benign from malignant liver masses with Acoustic Radiation Force Impulse technique. Ultrasound Q 2011;27:217-223.

26. Dong Y, Wang WP, Xu Y, Cao J, Mao F, Dietrich CF. Point shear wave speed measurement in differentiating benign and malignant focal liver lesions. Med Ultrason 2017;19:259-264.

27. Dietrich CF, Shi L, Wei Q, et al. What does liver elastography measure? Technical aspects and methodology. Minerva Gastroenterol Dietol 2020;doi: 10.23736/S1121421X.20.02787-7.

28. Dong Y, Sirli R, Ferraioli G, et al. Shear wave elastography of the liver - review on normal values. Z Gastroenterol 2017;55:153-166.

29. Barr RG, Wilson SR, Rubens D, Garcia-Tsao G, Ferraioli G. Update to the Society of Radiologists in Ultrasound Liver Elastography Consensus Statement. Radiology 2020;296:263-274.

30. Dietrich CF, Sirli R, Ferraioli G, et al. Current knowledge in ultrasound-based liver elastography of pediatric patients. Appl Sci 2018;8:944-963.

31. Ferraioli G, Barr RG, Dillman JR. Elastography for Pediatric Chronic Liver Disease: A Review and Expert Opinion. J Ultrasound Med 2020; doi: 10.1002/jum.15482.

32. Hall TJ, Milkowski A, Garra B, et al. RSNA/QIBA: shear wave speed as a biomarker for liver fibrosis staging. In: Ultrasonics Symposium (IUS), 2013 I.E. International; 2013;97-400.

33. Chang S, Kim MJ, Kim J, Lee MJ. Variability of shear wave velocity using different frequencies in acoustic radiation force impulse (ARFI) elastography: a phantom and normal liver study. Ultraschall Med 2013;34:260-265. 
34. Ling W, Lu Q, Quan J, Ma L, Luo Y. Assessment of impact factors on shear wave based liver stiffness measurement. Eur J Radiol 2013;82:335-341.

35. Ferraioli G, Lissandrin R, Zicchetti M, Bernuzzi S, Salvaneschi L, Filice C, Group ES. Ultrasound point shear wave elastography assessment of liver and spleen stiffness: effect of training on repeatability of measurements. Eur Radiol 2014;24:1283-1289.

36. D’Onofrio M, Gallotti A, Mucelli RP. Tissue quantification with acoustic radiation force impulse imaging: Measurement repeatability and normal values in the healthy liver. AJR Am J Roentgenol 2010;195:132-136.

37. Bota S, Sporea I, Sirli R, Popescu A, Jurchis A. Factors which influence the accuracy of acoustic radiation force impulse (ARFI) elastography for the diagnosis of liver fibrosis in patients with chronic hepatitis C. Ultrasound Med Biol 2013;39:407-412.

38. Ferraioli G, De Silvestri A, Reiberger T, et al. Adherence to quality criteria improves concordance between transient elastography and ElastPQ for liver stiffness assessment-A multicenter retrospective study. Dig Liver Dis 2018;50:1056-1061.

39. Ferraioli G, Maiocchi L, Lissandrin R, Tinelli C, De Silvestri A, Filice C, Liver Fibrosis Study G. Accuracy of the ElastPQ Technique for the Assessment of Liver Fibrosis in Patients with Chronic Hepatitis C: a "Real Life" Single Center Study. J Gastrointestin Liver Dis 2016;25:331-335.

40. Roccarina D, Iogna Prat L, Buzzetti E, et al. Establishing Reliability Criteria for Liver ElastPQ Shear Wave Elastography (ElastPQ-SWE): Comparison Between 10, 5 and 3 Measurements. Ultraschall Med 2019; doi: 10.1055/a-10106052.

41. Fang C, Jaffer OS, Yusuf GT, et al. Reducing the Number of Measurements in Liver Point Shear-Wave Elastography: Factors that Influence the Number and Reliability of Measurements in Assessment of Liver Fibrosis in Clinical Practice. Radiology 2018:172104.

42. Fang C, Jaffer OS, Yusuf GT, Konstantatou E, Quinlan DJ, Agarwal K, Quaglia A, et al. Reducing the Number of Measurements in Liver Point Shear-Wave Elastography: Factors that Influence the Number and Reliability of Measurements in Assessment of Liver Fibrosis in Clinical Practice. Radiology 2018;287:844-852.

43. Barr RG, Wilson SR, Rubens D, Garcia-Tsao G, Ferraioli G. Update to the Society of Radiologists in Ultrasound Liver Elastography Consensus Statement. Radiology 2020:192437.

44. Fraquelli M, Rigamonti C, Casazza G, et al. Reproducibility of transient elastography in the evaluation of liver fibrosis in patients with chronic liver disease. Gut 2007;56:968973.

45. Boursier J, Konate A, Gorea G, et al. Reproducibility of liver stiffness measurement by ultrasonographic elastometry. Clin Gastroenterol Hepatol 2008;6:1263-1269.

46. Afdhal NH, Bacon BR, Patel K, et al. Accuracy of fibroscan, compared with histology, in analysis of liver fibrosis in patients with hepatitis B or C: a United States multicenter study. Clin Gastroenterol Hepatol 2015;13:772-779 e771773.

47. Guzmán-Aroca F, Reus M, Berná-Serna JD, et al. Reproducibility of shear wave velocity measurements by acoustic radiation force impulse imaging of the liver: a study in healthy volunteers. J Ultrasound Med 2011;30:975-979.

48. Friedrich-Rust M, Wunder K, Kriener S, et al. Liver fibrosis in viral hepatitis: noninvasive assessment with acoustic radiation force impulse imaging versus transient elastography. Radiology 2009;252:595-604.

49. Bota S, Sporea I, Sirli R, Popescu A, Danila M, Costachescu D. Intra- and interoperator reproducibility of acoustic radiation force impulse (ARFI) elastography--preliminary results. Ultrasound Med Biol 2012;38:1103-1108.

50. Yoon JH, Lee JM, Han JK, Choi BI. Shear wave elastography for liver stiffness measurement in clinical sonographic examinations: evaluation of intraobserver reproducibility, technical failure, and unreliable stiffness measurements. J Ultrasound Med 2014;33:437-447.

51. Thiele M, Detlefsen S, Sevelsted Moller L, et al. Transient and 2-Dimensional Shear-Wave Elastography Provide Comparable Assessment of Alcoholic Liver Fibrosis and Cirrhosis. Gastroenterology 2016;150:123-133.

52. Cassinotto C, Boursier J, De Ledinghen V, et al. Liver stiffness in nonalcoholic fatty liver disease: A comparison of Supersonic Shear Imaging, FibroScan and ARFI with liver biopsy. Hepatology 2016;63:1817-1827.

53. Dietrich CF, Ferraioli G, Sirli R, et al. General advice in ultrasound based elastography of pediatric patients. Med Ultrason 2019;21:315-326.

54. Ferraioli G, De Silvestri A, Lissandrin R, et al. Evaluation of Inter-System Variability in Liver Stiffness Measurements. Ultraschall Med 2019;40:64-75.

55. Dietrich CF, Trenker C, Fontanilla T, et al. New Ultrasound Techniques Challenge the Diagnosis of Sinusoidal Obstruction Syndrome. Ultrasound Med Biol 2018;44:2171-2182.

56. de Franchis R, Baveno VIF. Expanding consensus in portal hypertension: Report of the Baveno VI Consensus Workshop: Stratifying risk and individualizing care for portal hypertension. J Hepatol 2015;63:743-752.

57. Bazerbachi F, Haffar S, Wang Z, et al. Range of Normal Liver Stiffness and Factors Associated With Increased Stiffness Measurements in Apparently Healthy Individuals. Clin Gastroenterol Hepatol 2019;17:54-64.e51.

58. Hodge A, Lim S, Goh E, et al. Coffee Intake Is Associated with a Lower Liver Stiffness in Patients with Non-Alcoholic Fatty Liver Disease, Hepatitis C, and Hepatitis B. Nutrients 2017;9:56.

59. Langdon JH, Elegbe E, Gonzalez RS, Osapoetra L, Ford T, McAleavey SA. Measurement of Liver Stiffness Using Shear Wave Elastography in a Rat Model: Factors Impacting Stiffness Measurement with Multiple- and Single-Tracking-Location Techniques. Ultrasound Med Biol 2017;43:2629-2639.

60. Coco B, Oliveri F, Maina AM, et al. Transient elastography: a new surrogate marker of liver fibrosis influenced by major changes of transaminases. J Viral Hepat 2007;14:360-369. 
61. Sagir A, Erhardt A, Schmitt M, Haussinger D. Transient elastography is unreliable for detection of cirrhosis in patients with acute liver damage. Hepatology 2008;47:592-595.

62. Arena U, Vizzutti F, Corti G, et al. Acute viral hepatitis increases liver stiffness values measured by transient elastography. Hepatology 2008;47:380-384.

63. Vigano M, Massironi S, Lampertico P, et al. Transient elastography assessment of the liver stiffness dynamics during acute hepatitis B. Eur J Gastroenterol Hepatol 2010;22:180184.

64. Millonig G, Reimann FM, Friedrich S, et al. Extrahepatic cholestasis increases liver stiffness (FibroScan) irrespective of fibrosis. Hepatology 2008;48:1718-1723.

65. Millonig G, Friedrich S, Adolf S, Fet al. Liver stiffness is directly influenced by central venous pressure. J Hepatol 2010;52:206-210.

66. Colli A, Pozzoni P, Berzuini A, et al. Decompensated chronic heart failure: increased liver stiffness measured by means of transient elastography. Radiology 2010;257:872-878.

67. Huang R, Gao ZH, Tang A, Sebastiani G, Deschenes M. Transient elastography is an unreliable marker of liver fibrosis in patients with portal vein thrombosis. Hepatology 2018;68:783-785.

68. Ferraioli G, Barr RG. Ultrasound liver elastography beyond liver fibrosis assessment. World J Gastroenterol 2020;26:3413-3420.

69. Krouskop TA, Wheeler TM, Kallel F, Garra BS, Hall T. Elastic moduli of breast and prostate tissues under compression. Ultrason Imaging 1998;20:260-274.

70. Cantisani V, David E, Barr RG, et al. US-Elastography for Breast Lesion Characterization: Prospective Comparison of US BIRADS, Strain Elastography and Shear wave Elastography. Ultraschall Med 2020; doi: 10.1055/a-1134-4937.

71. Barr RG. Sonographic breast elastography: a primer. J U1trasound Med 2012;31:773-783.

72. Barr RG. Comparison of Strain Elastography, Shearwave Elastography, and Shearwave Elastography with a Quality MEasure in Evaluation of Breast Masses. In: Texas Uo, editor. Eleventh International Tissue Elasticity Conference. Deauville, France: University of Texas; 2012:3-4.

73. Barr RG, De Silvestri A, Scotti V, et al. Diagnostic Performance and Accuracy of the 3 Interpreting Methods of Breast Strain Elastography: A Systematic Review and Meta-analysis. J Ultrasound Med 2019;38:1397-1404.

74. Barr RG, Zhang Z. Shear Wave Elastography of the Breast: Value of a Quality Measure and Comparison to Strain Elastography. Radiology 2015;275:45-53

75. Barr RG, Destounis S, Lackey LB, 2nd, Svensson WE, Balleyguier C, Smith C. Evaluation of breast lesions using sonographic elasticity imaging: a multicenter trial. J Ultrasound Med 2012;31:281-287.

76. Berg WA, Cosgrove DO, Dore CJ, et al. Shear-wave elastography improves the specificity of breast US: the BE1 multinational study of 939 masses. Radiology 2012;262:435-449.

77. Cosgrove DO, Berg WA, Dore CJ, et al. Shear wave elastography for breast masses is highly reproducible. Eur Radiol 2012;22:1023-1032.
78. Schafer FK, Hooley RJ, Ohlinger R, et al. ShearWave Elastography BE1 multinational breast study: additional SWE features support potential to downgrade BI-RADS(R)-3 lesions. Ultraschall Med 2013;34:254-259.

79. Maier AM, Heil J, Harcos A, et al. Prediction of pathological complete response in breast cancer patients during neoadjuvant chemotherapy: Is shear wave elastography a useful tool in clinical routine? Eur J Radiol 2020;128:109025.

80. Mendelson EB B-VM, Berg WA. ACR BI-RADS® Ultrasound in: ACR BI-RADS ${ }^{\circledR}$ Atlas, Breast Imaging Reporting and Data System. In: Reston, VA, American College of Radiology; 2013.

81. Zheng X, Huang Y, Liu Y, et al. Shear-Wave Elastography of the Breast: Added Value of a Quality Map in Diagnosis and Prediction of the Biological Characteristics of Breast Cancer. Korean J Radiol 2020;21:172-180.

82. Wojcinski S, Brandhorst K, Sadigh G, Hillemanns P, Degenhardt F. Acoustic radiation force impulse imaging with virtual touch tissue quantification: measurements of normal breast tissue and dependence on the degree of pre-compression. Ultrasound Med Biol 2013;39:2226-2232.

83. Tozaki M, Fukuma E. Pattern classification of ShearWave Elastography images for differential diagnosis between benign and malignant solid breast masses. Acta Radiol 2011;52:1069-1075.

84. Cong R, Li J, Guo S. A new qualitative pattern classification of shear wave elastograghy for solid breast mass evaluation. Eur J Radiol 2017;87:111-119.

85. Barr RG. Breast Elastography: How to Perform and Integrate Into a "Best-Practice" Patient Treatment Algorithm. J Ultrasound Med 2020;39:7-17.

86. Tanter M, Bercoff J, Athanasiou A, et al. Quantitative assessment of breast lesion viscoelasticity: initial clinical results using supersonic shear imaging. Ultrasound Med Biol 2008;34:1373-1386.

87. Dighe M, Barr R, Bojunga J, et al. Thyroid Ultrasound: State of the Art. Part 2 - Focal Thyroid Lesions. Med Ultrason 2017;19:195-210.

88. Dighe M, Barr R, Bojunga J, et al. Thyroid Ultrasound: State of the Art Part 1 - Thyroid Ultrasound reporting and Diffuse Thyroid Diseases. Med Ultrason 2017;19:7993.

89. Jiang M, Li C, Tang S, et al. Nomogram Based on ShearWave Elastography Radiomics Can Improve Preoperative Cervical Lymph Node Staging for Papillary Thyroid Carcinoma. Thyroid 2020;30:885-897.

90. Cantisani V, David E, Sidhu PS, et al. Parotid Gland Lesions: Multiparametric Ultrasound and MRI Features. Ultraschall Med 2016;37:454-471.

91. Fukuhara T, Matsuda E, Donishi R, et al. Clinical efficacy of novel elastography using acoustic radiation force impulse (ARFI) for diagnosis of malignant thyroid nodules. Laryngoscope Investig Otolaryngol 2018;3:319-325.

92. Cosgrove D, Barr R, Bojunga J, et al. WFUMB Guidelines and Recommendations on the Clinical Use of Ultrasound Elastography: Part 4. Thyroid. Ultrasound Med Biol 2017;43:4-26. 
93. Cantisani V, David E, Grazhdani H, et al. Prospective Evaluation of Semiquantitative Strain Ratio and Quantitative 2D Ultrasound Shear Wave Elastography (SWE) in Association with TIRADS Classification for Thyroid Nodule Characterization. Ultraschall Med 2019;40:495-503.

94. Bhatia KS, Cho CC, Tong CS, Lee YY, Yuen EH, Ahuja AT. Shear wave elastography of focal salivary gland lesions: preliminary experience in a routine head and neck US clinic. Eur Radiol 2012;22:957-965.

95. Badarinza M, Serban O, Maghear L, Bocsa C, Fodor D. Shear wave elastography as a new method to identify parotid lymphoma in primary Sjögren Syndrome patients: an observational study. Rheumatology International 2020;40:1275-1281.

96. Caliskan E, Ozturk M, Bayramoglu Z, Comert RG, Adaletli I. Evaluation of parotid glands in healthy children and adolescents using shear wave elastography and superb microvascular imaging. La Radiologia Medica 2018;123:1-9.

97. Badea AF, Tamas Szora A, Ciuleanu E, et al. ARFI quantitative elastography of the submandibular glands. Normal measurements and the diagnosis value of the method in radiation submaxillitis. Med Ultrason 2013;15:173-179.

98. Mandalia UY, Porte FN, Howlett DC. Salivary Gland: Oncologic Imaging. Ultrasound Clinics 2014;9:99-113.

99. Herman J, Sedlackova Z, Vachutka J, Furst T, Salzman R, Vomacka J. Shear wave elastography parameters of normal soft tissues of the neck. Biomed Pap Med Fac Univ Palacky Olomouc Czech Repub 2017;161:320-325.

100. Chen YL, Chang C, Zeng W, Wang F, Chen JJ, Qu N. 3-Dimensional shear wave elastography of breast lesions: Added value of color patterns with emphasis on crater sign of coronal plane. Medicine (Baltimore) 2016;95: e4877.

101. Tozaki M, Isobe S, Fukuma E. Preliminary study of ultrasonographic tissue quantification of the breast using the acoustic radiation force impulse (ARFI) technology. Eur J Radiol 2011;80:e182-187.

102. Zhou J, Zhan W, Chang C, et al. Breast lesions: evaluation with shear wave elastography, with special emphasis on the "stiff rim" sign. Radiology 2014;272:63-72. 